\title{
Static Embeddings as Efficient Knowledge Bases?
}

\author{
Philipp Dufter*, Nora Kassner*, Hinrich Schütze \\ Center for Information and Language Processing (CIS), LMU Munich, Germany \\ \{philipp, kassner\}@cis.lmu.de
}

\begin{abstract}
Recent research investigates factual knowledge stored in large pretrained language models (PLMs). Instead of structural knowledge base (KB) queries, masked sentences such as "Paris is the capital of [MASK]" are used as probes. The good performance on this analysis task has been interpreted as PLMs becoming potential repositories of factual knowledge. In experiments across ten linguistically diverse languages, we study knowledge contained in static embeddings. We show that, when restricting the output space to a candidate set, simple nearest neighbor matching using static embeddings performs better than PLMs. E.g., static embeddings perform $1.6 \%$ points better than BERT while just using $0.3 \%$ of energy for training. One important factor in their good comparative performance is that static embeddings are standardly learned for a large vocabulary. In contrast, BERT exploits its more sophisticated, but expensive ability to compose meaningful representations from a much smaller subword vocabulary.
\end{abstract}

\section{Introduction}

Pretrained language models (PLMs) (Peters et al., 2018; Howard and Ruder, 2018; Devlin et al., 2019) can be finetuned to a variety of natural language processing (NLP) tasks and then generally yield high performance. Increasingly, these models and their generative variants (e.g., GPT, Brown et al., 2020) are used to solve tasks by simple text generation, without any finetuning. This motivated research on how much knowledge is contained in PLMs: Petroni et al. (2019) used models pretrained with a masked language objective to answer clozestyle templates such as:

(Ex1) Paris is the capital of [MASK].

Using this methodology, Petroni et al. (2019) showed that PLMs capture some knowledge implicitly. This has been interpreted as suggesting

\footnotetext{
${ }^{*}$ Equal contribution - random order
}

\begin{tabular}{l|r|rr} 
Model & Vocabulary Size & \multicolumn{2}{|c}{ p1 } \\
& & LAMA & LAMA-UHN \\
\hline \hline Oracle & & 22.0 & 23.7 \\
\hline BERT & $30 \mathrm{k}$ & 39.6 & 30.7 \\
mBERT & $110 \mathrm{k}$ & 36.3 & 27.4 \\
\hline \multirow{6}{*}{ fastText } & BERT-30k & 26.9 & 16.8 \\
& mBERT-110k & 27.5 & 17.8 \\
& $30 \mathrm{k}$ & 16.4 & 5.8 \\
& $120 \mathrm{k}$ & 34.3 & 25.0 \\
& $250 \mathrm{k}$ & 37.7 & 29.0 \\
& $500 \mathrm{k}$ & 39.9 & 31.8 \\
& $1000 \mathrm{k}$ & $\mathbf{4 1 . 2}$ & $\mathbf{3 3 . 4}$
\end{tabular}

Table 1: Results for majority oracle, BERT, mBERT and fastText. Static fastText embeddings are competitive and outperform BERT for large vocabularies. BERT and mBERT use their subword vocabularies. For fastText, we use BERT/mBERT's vocabularies and newly trained wordpiece vocabularies on Wikipedia.

that PLMs are promising as repositories of factual knowledge. In this paper, we present evidence that simple static embeddings like fastText perform as well as PLMs in the context of answering knowledge base (KB) queries. Answering KB queries can be decomposed into two subproblems, typing and ranking. Typing refers to the problem of predicting the correct type of the answer entity; e.g., "country" is the correct type for [MASK] in (Ex1), a task that PLMs seem to be good at. Ranking consists of finding the entity of the correct type that is the best fit ("France" in (Ex1)). By restricting the output space to the correct type we disentangle the two subproblems and only evaluate ranking. We do this for three reasons. (i) Ranking is the knowledgeintensive step and thus the key research question. (ii) Typed querying reduces PLMs' dependency on the template. (iii) It allows a direct comparison between static word embeddings and PLMs. Prior work has adopted a similar approach (Xiong et al., 2020; Kassner et al., 2021).

For a PLM like BERT, ranking amounts to finding the entity whose embedding is most similar 
to the output embedding for [MASK]. For static embeddings, we rank entities (e.g., entities of type country) with respect to similarity to the query entity (e.g., "Paris" in (Ex1)). In experiments across ten linguistically diverse languages, we show that this simple nearest neighbor matching with fastText embeddings performs comparably to or even better than BERT. For example for English, fastText embeddings perform $1.6 \%$ points better than BERT ( $41.2 \%$ vs. $39.6 \%$, see Table 1, column "LAMA"). This suggests that BERT's core mechanism for answering factual queries is not more effective than simple nearest neighbor matching using fastText embeddings.

We believe this means that claims that PLMs are KBs have to be treated with caution. Advantages of BERT are that it composes meaningful representations from a small subword vocabulary and handles typing implicitly (Petroni et al., 2019). In contrast, answering queries without restricting the answer space to a list of candidates is hard to achieve with static word embeddings. On the other hand, static embeddings are cheap to obtain, even for large vocabulary sizes. This has important implications for green NLP. PLMs require tremendous computational resources, whereas static embeddings have only $0.3 \%$ of the carbon footprint of BERT (see Table 4). This argues for proponents of resourcehungry deep learning models to try harder to find cheap "green" baselines or to combine the best of both worlds (cf. Poerner et al., 2020).

In summary, our contributions are:

i) We propose an experimental setup that allows a direct comparison between PLMs and static word embeddings. We find that static word embeddings show performance similar to BERT on the modified LAMA analysis task across ten languages.

ii) We provide evidence that there is a trade-off between composing meaningful representations from subwords and increasing the vocabulary size. Storing information through composition in a network seems to be more expensive and challenging than simply increasing the number of atomic representations.

iii) Our findings may point to a general problem: baselines that are simpler and "greener" are not given enough attention in deep learning.

Code and embeddings are available online. ${ }^{1}$

\footnotetext{
${ }^{1}$ https://github.com/pdufter/staticlama
}

\begin{tabular}{llll} 
Language & Code & Family & Script \\
\hline \hline Arabic & AR & Afro-Asiatic & Arabic \\
German & DE & Indo-European & Latin \\
English & EN & Indo-European & Latin \\
Spanish & ES & Indo-European & Latin \\
Finnish & FI & Uralic & Latin \\
Hebrew & HE & Afro-Asiatic & Hebrew \\
Japanese & JA & Japonic & Japanese \\
Korean & KO & Koreanic & Korean \\
Turkish & TR & Turkic & Latin \\
Thai & TH & Tai-Kadai & Thai
\end{tabular}

Table 2: Overview of the ten languages in our experiments, including language family and script.

\section{Data}

We follow the LAMA setup introduced by Petroni et al. (2019). More specifically, we use data from TREx (Elsahar et al., 2018). TREx consists of triples of the form (object, relation, subject). The underlying idea of LAMA is to query knowledge from PLMs using templates without any finetuning: the triple (Paris, capital-of, France) is queried with the template "Paris is the capital of [MASK]." TREx covers 41 relations. Templates for each relation were manually created by Petroni et al. (2019). LAMA has been found to contain many "easy-toguess" triples; e.g., it is easy to guess that a person with an Italian sounding name is Italian. LAMAUHN is a subset of triples that are "hard-to-guess" created by Poerner et al. (2020).

Beyond English, we run experiments on nine additional languages using mLAMA, a multilingual version of TREx (Kassner et al., 2021). For an overview of languages and language families see Table 2. For training static embeddings, we use Wikipedia dumps from October 2020.

\section{Methods}

We describe our proposed setup, which allows to compare PLMs with static embeddings.

\subsection{PLMs}

We use the following two PLMs: (i) BERT for English (BERT-base-cased, Devlin et al. (2019)), (ii) mBERT for all ten languages (the multilingual version BERT-base-multilingual-cased).

Petroni et al. (2019) use templates like "Paris is the capital of [MASK]" and give $\arg \max _{w \in \mathcal{V}} p(w \mid t)$ as answer where $\mathcal{V}$ is the vocabulary of the PLM and $p(w \mid t)$ is the probability that word $w$ gets predicted in the template $t$.

We follow the same setup as (Kassner et al., 


\begin{tabular}{lrrrrrrrrrr} 
Model & $\begin{array}{r}\text { Vocab. } \\
\text { Size }\end{array}$ & AR & DE & ES & FI & HE & JA & KO & TH & TR \\
\hline \hline Oracle & & 21.9 & 22.3 & 21.6 & 21.3 & 22.9 & 21.3 & 21.7 & 23.7 & 23.5 \\
\hline mBERT & $110 \mathrm{k}$ & 17.2 & 31.5 & 33.6 & 20.6 & 17.5 & 15.1 & 18.9 & 13.5 & 33.8 \\
\hline & mB-110k & 16.4 & 20.9 & 24.6 & 21.4 & 14.5 & 12.9 & 16.1 & 12.9 & 26.0 \\
& $30 \mathrm{k}$ & 20.8 & 16.2 & 17.1 & 16.7 & 21.4 & 14.6 & 17.3 & 21.3 & 22.1 \\
fastText & $120 \mathrm{k}$ & 27.9 & 25.2 & 31.0 & 24.2 & 28.3 & 22.4 & 28.2 & 28.0 & 33.2 \\
& $250 \mathrm{k}$ & 30.1 & 30.3 & 34.2 & 28.8 & 32.8 & 24.9 & 30.5 & 31.6 & 35.6 \\
& 500k & $\mathbf{3 1 . 7}$ & 32.5 & $\mathbf{3 6 . 6}$ & 30.9 & 33.7 & 27.0 & $\mathbf{3 1 . 5}$ & $\mathbf{3 1 . 8}$ & 36.1 \\
& $1000 \mathrm{k}$ & 31.3 & $\mathbf{3 3 . 6}$ & 36.5 & $\mathbf{3 1 . 8}$ & $\mathbf{3 3 . 9}$ & $\mathbf{2 7 . 2}$ & 29.8 & 30.5 & $\mathbf{3 6 . 6}$
\end{tabular}

Table 3: $\mathrm{p} 1$ for mBERT and fastText on mLAMA. fastText clearly outperforms mBERT for large vocabularies. Numbers across languages are not comparable as the number of triples varies.

\begin{tabular}{lrrrr} 
Model & Power $(\mathbf{W})$ & $\mathbf{h}$ & $\mathbf{k W h} \cdot \mathbf{P U E}$ & $\mathbf{C O}_{\mathbf{2}} \mathbf{e}$ \\
\hline \hline BERT & 12,041 & 79 & 1,507 & 1,438 \\
fastText-en & 618 & 5 & 5 & 5 \\
\hline ratio-en & 0.05 & 0.06 & 0.003 & 0.003
\end{tabular}

Table 4: Power consumption (Power), hours of computation (h), energy consumption (kWh - PUE) and carbon emissions $\left(\mathrm{CO}_{2} \mathrm{e}\right)$ of BERT vs. fastText. Training embeddings for all languages takes around 4 times the resources as training English. BERT numbers from (Strubell et al., 2019). We use our server's peak power consumption. See appendix for details.

2021) and use typed querying: for each relation, we create a candidate set $\mathcal{C}$ and then predict $\arg \max _{c \in \mathcal{C}} p(c \mid t)$. For most templates, there is only one valid entity type, e.g., country for (Ex1). We choose as $\mathcal{C}$ the set of objects across all triples for a single relation. The candidate set could also be obtained from an entity typing system (e.g., Yaghoobzadeh et al., 2018), but this is beyond the scope of this paper. Variants of typed prediction have been used before (Xiong et al., 2020).

We accommodate multi-token objects, i.e., objects that are not contained in the vocabulary, by including multiple [MASK] tokens in the templates. We then compute an object's score as the average of the $\log$ probabilities for its individual tokens. Note that we do not perform any finetuning.

\subsection{Vocabulary}

The vocabulary $\mathcal{V}$ of the wordpiece tokenizer is of central importance for static embeddings as well as PLMs. BERT models come with fixed vocabularies. It would be prohibitive to retrain the models with a new vocabulary. It would also be too expensive to increase the vocabulary by a large factor: the embedding matrix is responsible for the majority of the memory consumption of these models.

In contrast, increasing the vocabulary size is cheap for static embeddings. We thus experiment with different vocabulary sizes for static embeddings. To this end, we train new vocabularies for each language on Wikipedia using the wordpiece tokenizer (Schuster and Nakajima, 2012).

\subsection{Static Embeddings}

Using either newly trained vocabularies or existing BERT vocabularies, we tokenize Wikipedia. We then train fastText embeddings (Bojanowski et al., 2017) with default parameters (http://fasttext.cc). We consider the same candidate set $\mathcal{C}$ as for PLMs. Let $c \in \mathcal{C}$ be a candidate that gets split into tokens $t_{1}, \ldots, t_{k}$ by the wordpiece tokenizer. We then assign to $c$ the embedding vector

$$
\bar{e}_{c}=\frac{1}{k} \sum_{i=1}^{k} e_{t_{i}}
$$

where $e_{t_{i}}$ is the fastText vector for token $t_{i}$. We compute the representations for a query $q$ analogously. For a query $q$ (the subject of a triple), we then compute the prediction as:

$$
\arg \max _{c \in \mathcal{C}} \operatorname{cosine}-\operatorname{sim}\left(\bar{e}_{q}, \bar{e}_{c}\right),
$$

i.e., we perform simple nearest neighbor matching. Note that the static embedding method does not get any signal about the relation. The method's only input is the subject of a triple, and we leave incorporating a relation vector to future work.

\subsection{Evaluation Metric}

We compute precision at one for each relation, i.e., $1 /|T| \sum_{t \in T} \mathbb{1}\left\{\hat{t}_{\text {object }}=t_{\text {object }}\right\}$ where $T$ is the set of all triples and $\hat{t}_{\text {object }}$ the object predicted using contextualized/static embeddings. Note that $T$ is different for each language. Our final measure (p1) is then the precision at one (macro-)averaged over relations. As a consistency check we provide an Oracle baseline: it always predicts the most frequent object across triples based on the gold candidate sets.

\section{Results and Discussion}

In this section, we compare the performance of BERT and fastText, analyze their resource consumption, and give evidence that BERT composes meaningful representations from subwords. 


\subsection{BERT vs. fastText}

Results for English are in Table 1. The table shows that when increasing the vocabulary size, static embeddings and BERT exhibit similar performance on LAMA. The Oracle baseline is mostly outperformed. Only for small vocabulary sizes, fastText is worse. Performance of fastText increases with larger vocabulary sizes and with a vocabulary size of $1000 \mathrm{k}$ we observe a $1.6 \%$ absolute performance increase of fastText embeddings compared to BERT $(41.2 \%$ vs. $39.6 \%)$. The performance gap between fastText and BERT increases to $2.7 \%$ points on LAMA-UHN, indicating that fastText is less vulnerable to misleading clues about the subject.

Only providing results on English can be prone to unexpected biases. Thus, we verify our results for nine additional languages. Results are shown in Table 3 and the conclusions are similar: for large enough vocabularies, static embeddings consistently have better performance. For languages outside the Indo-European family, the performance gap between mBERT and fastText is much larger (e.g., 31.7 vs. 17.2 for Arabic) and mBERT is sometimes worse than the Oracle.

Our fastText method is quite primitive: it is a type-restricted search for entities similar to what is most prominent in the context (whose central element is the query entity, e.g., "Paris" in (Ex1)). The fact that fastText outperforms BERT raises the question: Does BERT simply use associations between entities (like fastText) or has it captured factual knowledge beyond this?

\subsection{BERT vs fastText: Diversity of Predictions}

The entropy of the distribution of predicted objects is 6.5 for BERT vs. 7.3 for fastText. So BERT's predictions are less diverse. Of 151 possible objects on average, BERT predicts (on average) 85, fastText 119. For a given relation, BERT's prediction tend to be dominated by one object, which is often the most frequent correct object - possibly because these objects are frequent in Wikipedia/Wikidata. When filtering out triples whose correct answer is the most frequent object, BERT's performance drops to 35.7 whereas fastText's increases to 42.5 . See Table 7 in the appendix for full results on diversity. We leave investigating why BERT has these narrower object preferences for future work.
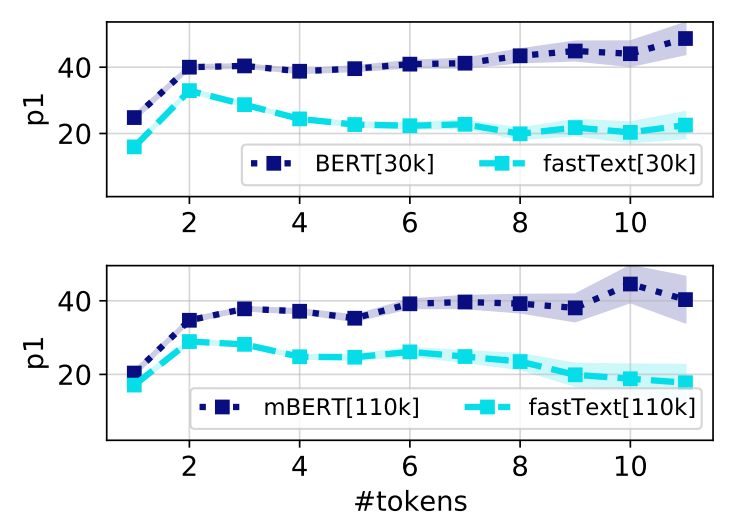

Figure 1: p1 as a function of the tokenization length of the triples' subjects. BERT and fastText use the same vocabulary here, ensuring comparability. BERT based models exhibit a stable performance independent of the number of tokens a subject gets split into. In contrast, fastText's performance drops.

\subsection{Contextualization in BERT}

BERT's attention mechanism should be able to handle long subjects - in contrast to fastText, for which we use simple averaging. Figure 1 shows that fastText's performance indeed drops when the query gets tokenized into multiple tokens. In contrast, BERT's performance remains stable. We conclude that token averaging harms fastText's performance and that the attention mechanism in BERT composes meaningful representations from subwords.

We try to induce static embeddings from BERT by feeding object and subject surface forms to BERT without any context and then averaging the hidden representations for each layer. Figure 2 analyzes whether a nearest neighbor matching over this static embedding space extracted from BERT's representations is effective in extracting knowledge from it. We find that performance on LAMA is significantly lower across all hidden layers with the first two layers performing best. That simple averaging does not work as well as contextualization indicates that BERT is great at composing meaningful representations through attention. In future work, it would be interesting to extract better static representations from BERT, for example by extracting the representations of entities in real sentences.

\subsection{Resource Consumption}

Table 4 compares resource consumption of BERT vs. fastText following Strubell et al. (2019). fastText can be efficiently computed on CPUs with a drastically lower power consumption and computation time. Overall, fastText has only $0.3 \%$ of the 


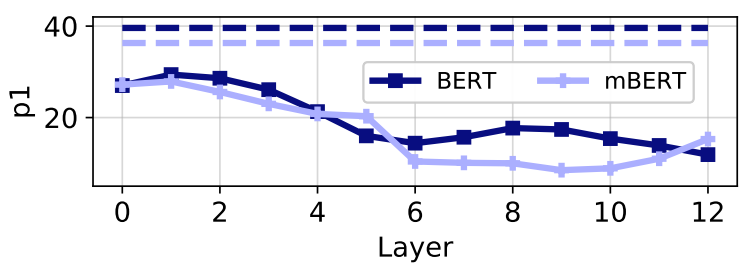

Figure 2: Contextualization in BERT. The dashed lines are p1 when querying with templates like "Paris is the capital of [MASK]." and a candidate set. The solid lines reflect performance of nearest neighbor matching with cosine similarity when inducing a static embedding space from the representations at these layers. This shows that extracting high quality static embeddings is not trivial, and BERT's contextualization is essential for getting good performance.

carbon emissions compared to BERT. In a recent study, Zhang et al. (2020) showed that capturing factual knowledge inside PLMs is an especially resource hungry task.

These big differences demonstrate that fastText, in addition to performing better than BERT, is the environmentally better model to "encode knowledge" of Wikipedia in an unsupervised fashion. This calls into question the use of large PLMs as knowledge bases, particularly in light of the recent surge of knowledge augmented LMs, e.g., (Lewis et al., 2020; Guu et al., 2020).

\section{Related Work}

Petroni et al. (2019) first asked: can PLMs function as KBs? Subsequent analysis focused on different aspects, such as negation (Kassner and Schütze, 2020; Ettinger, 2020), paraphrases (Elazar et al., 2021), easy to guess names (Poerner et al., 2020), finding alternatives to a cloze-style approach (Bouraoui et al., 2020; Heinzerling and Inui, 2020; Jiang et al., 2020) or analyzing different model sizes (Roberts et al., 2020).

There is a recent surge of work that tries to improve PLMs' ability to harvest factual knowledge: Zhang et al. (2019), Peters et al. (2019) and Wang et al. (2020) inject factual knowledge into PLMs. Guu et al. (2020), Lewis et al. (2020), Izacard and Grave (2020), Kassner and Schütze (2020) and Petroni et al. (2020) combine PLMs with information retrieval and Bosselut et al. (2019), Liu et al. (2020) and Yu et al. (2020) with knowledge bases.

In contrast, we provide evidence that BERT's ability to answer factual queries is not more effective than capturing "knowledge" with simple traditional static embeddings. This suggests that learning associations between entities and typerestricted similarity search over these associations may be at the core of BERT's ability to answer cloze-style KB queries, a new insight into BERT's working mechanism.

\section{Conclusion}

We have shown that, when restricting cloze-style questions to a candidate set, static word embeddings outperform BERT. To explain this puzzling superiority of a much simpler model, we put forward a new characterization of factual knowledge learned by BERT: BERT seems to be able to complete cloze-style queries based on similarity assessments on a type-restricted vocabulary much like a nearest neighbor search for static embeddings.

However, BERT may still be the better model for the task: we assume perfect typing (for BERT and fastText) and only evaluate ranking. Typing is much harder with static embeddings and BERT has been shown to perform well at guessing the expected entity type based on a template. BERT also works well with small vocabularies, storing most of its "knowledge" in the parameterization of subword composition. Our results suggest that increasing the vocabulary size and computing more atomic entity representations with fastText is a cheap and environmentally friendly method of storing knowledge. In contrast, learning high quality composition of smaller units requires many more resources.

fastText is a simple cheap baseline that outperforms BERT on LAMA, but was not considered in the original research. This may be an example of a general problem: "green" baselines are often ignored, but should be considered when evaluating resource-hungry deep learning models. A promising way forward would be to combine the best of both worlds, e.g., by building on work that incorporates large vocabularies into PLMs after pretraining.

Acknowledgements. This work was supported by the European Research Council (\# 740516) and the German Federal Ministry of Education and Research (BMBF) under Grant No. 01IS18036A. The authors of this work take full responsibility for its content. The first author was supported by the Bavarian research institute for digital transformation (bidt) through their fellowship program. We thank Yanai Elazar and the anonymous reviewers for valuable comments. 


\section{References}

Piotr Bojanowski, Edouard Grave, Armand Joulin, and Tomás Mikolov. 2017. Enriching word vectors with subword information. Trans. Assoc. Comput. Linguistics, 5:135-146.

Antoine Bosselut, Hannah Rashkin, Maarten Sap, Chaitanya Malaviya, Asli Celikyilmaz, and Yejin Choi. 2019. COMET: commonsense transformers for automatic knowledge graph construction. In Proceedings of the 57th Conference of the Association for Computational Linguistics, ACL 2019, Florence, Italy, July 28-August 2, 2019, Volume 1: Long Papers, pages 4762-4779. Association for Computational Linguistics.

Zied Bouraoui, José Camacho-Collados, and Steven Schockaert. 2020. Inducing relational knowledge from BERT. In The Thirty-Fourth AAAI Conference on Artificial Intelligence, AAAI 2020, The ThirtySecond Innovative Applications of Artificial Intelligence Conference, IAAI 2020, The Tenth AAAI Symposium on Educational Advances in Artificial Intelligence, EAAI 2020, New York, NY, USA, February 7-12, 2020, pages 7456-7463. AAAI Press.

Tom B. Brown, Benjamin Mann, Nick Ryder, Melanie Subbiah, Jared Kaplan, Prafulla Dhariwal, Arvind Neelakantan, Pranav Shyam, Girish Sastry, Amanda Askell, Sandhini Agarwal, Ariel Herbert-Voss, Gretchen Krueger, Tom Henighan, Rewon Child, Aditya Ramesh, Daniel M. Ziegler, Jeffrey Wu, Clemens Winter, Christopher Hesse, Mark Chen, Eric Sigler, Mateusz Litwin, Scott Gray, Benjamin Chess, Jack Clark, Christopher Berner, Sam McCandlish, Alec Radford, Ilya Sutskever, and Dario Amodei. 2020. Language models are few-shot learners. In Advances in Neural Information Processing Systems 33: Annual Conference on Neural Information Processing Systems 2020, NeurIPS 2020, December 6-12, 2020, virtual.

Jacob Devlin, Ming-Wei Chang, Kenton Lee, and Kristina Toutanova. 2019. BERT: Pre-training of deep bidirectional transformers for language understanding. In Proceedings of the 2019 Conference of the North American Chapter of the Association for Computational Linguistics: Human Language Technologies, Volume 1 (Long and Short Papers), pages 4171-4186, Minneapolis, Minnesota. Association for Computational Linguistics.

Yanai Elazar, Nora Kassner, Shauli Ravfogel, Abhilasha Ravichander, E. Hovy, H. Schutze, and Yoav Goldberg. 2021. Measuring and improving consistency in pretrained language models. ArXiv, abs/2102.01017.

Hady Elsahar, Pavlos Vougiouklis, Arslen Remaci, Christophe Gravier, Jonathon Hare, Frederique Laforest, and Elena Simperl. 2018. T-REx: A large scale alignment of natural language with knowledge base triples. In Proceedings of the Eleventh International Conference on Language Resources and
Evaluation (LREC-2018), Miyazaki, Japan. European Languages Resources Association (ELRA).

Allyson Ettinger. 2020. What BERT is not: Lessons from a new suite of psycholinguistic diagnostics for language models. Trans. Assoc. Comput. Linguistics, 8:34-48.

Kelvin Guu, Kenton Lee, Zora Tung, Panupong Pasupat, and Ming-Wei Chang. 2020. REALM: retrievalaugmented language model pre-training. Computing Research Repository, abs/2002.08909.

Benjamin Heinzerling and Kentaro Inui. 2020. Language models as knowledge bases: On entity representations, storage capacity, and paraphrased queries. Computing Research Repository, abs/2008.09036.

Jeremy Howard and Sebastian Ruder. 2018. Universal language model fine-tuning for text classification. In Proceedings of the 56th Annual Meeting of the Association for Computational Linguistics (Volume 1: Long Papers), pages 328-339, Melbourne, Australia. Association for Computational Linguistics.

Gautier Izacard and Edouard Grave. 2020. Leveraging passage retrieval with generative models for open domain question answering. Computing Research Repository, abs/2007.01282.

Zhengbao Jiang, Frank F. Xu, Jun Araki, and Graham Neubig. 2020. How can we know what language models know. Trans. Assoc. Comput. Linguistics, $8: 423-438$.

Nora Kassner, Philipp Dufter, and Hinrich Schütze. 2021. Multilingual LAMA: Investigating knowledge in multilingual pretrained language models. In Proceedings of the 16th Conference of the European Chapter of the Association for Computational Linguistics: Main Volume, pages 3250-3258, Online. Association for Computational Linguistics.

Nora Kassner and Hinrich Schütze. 2020. Bert-knn: Adding a knn search component to pretrained language models for better QA. In Proceedings of the 2020 Conference on Empirical Methods in Natural Language Processing: Findings, EMNLP 2020, Online Event, 16-20 November 2020, pages 3424-3430. Association for Computational Linguistics.

Nora Kassner and Hinrich Schütze. 2020. Negated and misprimed probes for pretrained language models: Birds can talk, but cannot fly. In Proceedings of the 58th Annual Meeting of the Association for Computational Linguistics, pages 7811-7818, Online. Association for Computational Linguistics.

Mike Lewis, Marjan Ghazvininejad, Gargi Ghosh, Armen Aghajanyan, Sida Wang, and Luke Zettlemoyer. 2020. Pre-training via paraphrasing. In Advances in Neural Information Processing Systems 33: Annual Conference on Neural Information Processing Systems 2020, NeurIPS 2020, December 6-12, 2020, virtual. 
Weijie Liu, Peng Zhou, Zhe Zhao, Zhiruo Wang, Qi Ju, Haotang Deng, and Ping Wang. 2020. KBERT: enabling language representation with knowledge graph. In The Thirty-Fourth AAAI Conference on Artificial Intelligence, AAAI 2020, The ThirtySecond Innovative Applications of Artificial Intelligence Conference, IAAI 2020, The Tenth AAAI Symposium on Educational Advances in Artificial Intelligence, EAAI 2020, New York, NY, USA, February 7-12, 2020, pages 2901-2908. AAAI Press.

Matthew Peters, Mark Neumann, Mohit Iyyer, Matt Gardner, Christopher Clark, Kenton Lee, and Luke Zettlemoyer. 2018. Deep contextualized word representations. In Proceedings of the 2018 Conference of the North American Chapter of the Association for Computational Linguistics: Human Language Technologies, Volume 1 (Long Papers), pages 2227-2237, New Orleans, Louisiana. Association for Computational Linguistics.

Matthew E. Peters, Mark Neumann, Robert Logan, Roy Schwartz, Vidur Joshi, Sameer Singh, and Noah A Smith. 2019. Knowledge enhanced contextual word representations. In Proceedings of the 2019 Conference on Empirical Methods in Natural Language Processing and the 9th International Joint Conference on Natural Language Processing (EMNLPIJCNLP), pages 43-54, Hong Kong, China. Association for Computational Linguistics.

Fabio Petroni, Patrick S. H. Lewis, Aleksandra Piktus, Tim Rocktäschel, Yuxiang Wu, Alexander H. Miller, and Sebastian Riedel. 2020. How context affects language models' factual predictions. In Conference on Automated Knowledge Base Construction, AKBC 2020, Virtual, June 22-24, 2020.

Fabio Petroni, Tim Rocktäschel, Sebastian Riedel, Patrick S. H. Lewis, Anton Bakhtin, Yuxiang Wu, and Alexander H. Miller. 2019. Language models as knowledge bases? In Proceedings of the 2019 Conference on Empirical Methods in Natural Language Processing and the 9th International Joint Conference on Natural Language Processing, EMNLP-IJCNLP 2019, Hong Kong, China, November 3-7, 2019, pages 2463-2473. Association for Computational Linguistics.

Nina Poerner, Ulli Waltinger, and Hinrich Schütze. 2020. E-BERT: Efficient-yet-effective entity embeddings for BERT. In Findings of the Association for Computational Linguistics: EMNLP 2020, pages 803-818, Online. Association for Computational Linguistics.

Adam Roberts, Colin Raffel, and Noam Shazeer. 2020. How much knowledge can you pack into the parameters of a language model? In Proceedings of the 2020 Conference on Empirical Methods in Natural Language Processing, EMNLP 2020, Online, November 16-20, 2020, pages 5418-5426. Association for Computational Linguistics.
Mike Schuster and Kaisuke Nakajima. 2012. Japanese and korean voice search. In 2012 IEEE International Conference on Acoustics, Speech and Signal Processing, ICASSP 2012, Kyoto, Japan, March 2530, 2012, pages 5149-5152. IEEE.

Emma Strubell, Ananya Ganesh, and Andrew McCallum. 2019. Energy and policy considerations for deep learning in NLP. In Proceedings of the 57th Annual Meeting of the Association for Computational Linguistics, pages 3645-3650, Florence, Italy. Association for Computational Linguistics.

Ruize Wang, Duyu Tang, Nan Duan, Zhongyu Wei, Xuanjing Huang, Jianshu Ji, Guihong Cao, Daxin Jiang, and Ming Zhou. 2020. K-adapter: Infusing knowledge into pre-trained models with adapters. Computing Research Repository, abs/2002.01808.

Wenhan Xiong, Jingfei Du, William Yang Wang, and Veselin Stoyanov. 2020. Pretrained encyclopedia: Weakly supervised knowledge-pretrained language model. In 8th International Conference on Learning Representations, ICLR 2020, Addis Ababa, Ethiopia, April 26-30, 2020. OpenReview.net.

Yadollah Yaghoobzadeh, Heike Adel, and Hinrich Schütze. 2018. Corpus-level fine-grained entity typing. J. Artif. Intell. Res., 61:835-862.

Donghan Yu, Chenguang Zhu, Yiming Yang, and Michael Zeng. 2020. JAKET: joint pre-training of knowledge graph and language understanding. Computing Research Repository, abs/2010.00796.

Yian Zhang, Alex Warstadt, Haau-Sing Li, and Samuel R. Bowman. 2020. When do you need billions of words of pretraining data? Computing Research Repository, abs/2011.04946.

Zhengyan Zhang, Xu Han, Zhiyuan Liu, Xin Jiang, Maosong Sun, and Qun Liu. 2019. ERNIE: enhanced language representation with informative entities. In Proceedings of the 57th Conference of the Association for Computational Linguistics, ACL 2019, Florence, Italy, July 28-August 2, 2019, Volume 1: Long Papers, pages 1441-1451. Association for Computational Linguistics. 


\section{A Resource Consumption}

We follow Strubell et al. (2019) for our computation. The measured peak energy consumption of our CPU-server was $618 \mathrm{~W}$. Considering the power usage effectiveness the required $\mathrm{kWh}$ are given by $p_{t}=1.58 \cdot t \cdot 618 / 1000$. Training the English fastText on Wikipedia took around 5 hours. Training all languages took 20 hours. The estimated $\mathrm{CO}_{2} \mathrm{e}$ can then be computed by $\mathrm{CO}_{2} \mathrm{e}=0.954 \cdot p_{t}$

\section{B Reproducibility Information}

For computation we use a CPU server with 96 CPU cores (Intel(R) Xeon(R) Platinum 8160) and 1024GB RAM. For BERT and mBERT inference we use a single GeForce GTX 1080Ti GPU.

Getting the object predictions for BERT and fastText is fast and takes a negligible amount of time. Training fastText embeddings takes between 1 to 5 hours depending on Wikipedia size.

BERT has around $110 \mathrm{M}$ parameters, mBERT around $178 \mathrm{M}$. The fastText embeddings have $\mathcal{O}(n d)$ parameters where $n$ is the vocabulary size and $d$ is the embedding dimension. We use $d=$ 300 . Thus, for most vocabulary sizes, fastText has significantly more parameters than the BERT models. But overall they are cheaper to train.

We did not perform any hyperparameter tuning. Table 6 gives an overview on third party software. Table 5 gives an overview on the number of triples in the dataset. Note that no training set is required, as all methods are completely unsupervised.

\section{Examples}

Table 11 shows randomly sampled triples to perform an error analysis.

\begin{tabular}{crr} 
Language & \#Triples & \#Triples UHN \\
\hline \hline ar & 17129 & 13699 \\
de & 29354 & 23493 \\
en & 33981 & 27060 \\
es & 28169 & 22683 \\
fr & 30643 & 24487 \\
he & 14769 & 12033 \\
ja & 22920 & 17832 \\
ko & 14217 & 11439 \\
th & 8327 & 7065 \\
tr & 13993 & 11274
\end{tabular}

Table 5: Overview on number of triples.

\begin{tabular}{lll} 
System & Parameter & Value \\
\hline \hline \multirow{2}{*}{ fastText } & Facebook Research & Version0.9.1 \\
BERT & Embedding Dimension & 300 \\
Tokenizers & Huggingface Transformer & Version 2.8.0 \\
& Huggingface Tokenizers & Version 0.5.2
\end{tabular}

Table 6: Overview on third party software.

Model Vocabulary Size p1 p1-mf entropy \#pred.

\begin{tabular}{|c|c|c|c|c|c|}
\hline Oracle & & 22.0 & 0.0 & 3.68 & 1 \\
\hline BERT & $30 \mathrm{k}$ & 39.6 & 35.7 & 6.48 & 85 \\
\hline mBERT & $110 \mathrm{k}$ & 36.3 & 32.6 & 6.41 & 86 \\
\hline \multirow{7}{*}{ fastText } & BERT-30k & 26.9 & 27.7 & 7.04 & 107 \\
\hline & nBERT-110k & 27.5 & 27.6 & 7.09 & 110 \\
\hline & $30 \mathrm{k}$ & 16.4 & 15.9 & 7.13 & 111 \\
\hline & $120 \mathrm{k}$ & 34.3 & 35.4 & 7.30 & 115 \\
\hline & $250 \mathrm{k}$ & 37.7 & 38.9 & 7.33 & 118 \\
\hline & $500 \mathrm{k}$ & 39.9 & 41.2 & 7.33 & 119 \\
\hline & $1000 \mathrm{k}$ & 41.2 & 42.5 & 7.32 & 119 \\
\hline
\end{tabular}

Table 7: Analysis of the diversity of predictions. $p 1-m f$ is the $\mathrm{p} 1$ when excluding triples whose correct answer is the most frequent object. entropy is the entropy of the distribution of predicted objects. \#pred. denotes the average number of distinct objects predicted by the model across relations. The average number of unique objects in the candidate set across relations is 151 . fastText has more diverse predictions, as the entropy is higher and the set of predicted objects is on average much larger.

\section{Additional Results}

In this section we show additional results. Table 8 shows the same as Table 1 but with precision at five. Analogously Table 9. Table 10 shows the same as Table 3 but for LAMA-UHN. The trends and key insights are unchanged. Table 7 analyses the diversity of predictions by the different models.

\begin{tabular}{lrrr} 
Model & Vocabulary Size & \multicolumn{2}{c}{ p5 } \\
& & LAMA & LAMA-UHN \\
\hline \hline Oracle & & 48.0 & 49.7 \\
\hline BERT & $30 \mathrm{k}$ & 64.1 & 57.9 \\
mBERT & $110 \mathrm{k}$ & 59.7 & 53.5 \\
\hline & BERT-30k & 48.7 & 41.9 \\
& mBERT-110k & 48.9 & 42.0 \\
& $30 \mathrm{k}$ & 26.3 & 16.5 \\
fastText & $120 \mathrm{k}$ & 58.3 & 52.7 \\
& $250 \mathrm{k}$ & 62.7 & 58.1 \\
& $500 \mathrm{k}$ & 65.4 & 61.3 \\
& $1000 \mathrm{k}$ & $\mathbf{6 6 . 8}$ & $\mathbf{6 3 . 1}$
\end{tabular}

Table 8: Results for BERT, mBERT and fastText. Same as Table 1 but with $\mathrm{p} 5$. 


\begin{tabular}{lrlllllllll} 
Model & $\begin{array}{c}\text { Vocab. } \\
\text { Size }\end{array}$ & AR & DE & ES & FI & HE & JA & KO & TH & TR \\
\hline \hline Oracle & 48.8 & 48.4 & 48.6 & 49.6 & 50.1 & 49.0 & 49.2 & 51.9 & 50.3 \\
\hline mBERT & 110k & 33.8 & 51.3 & 53.9 & 46.2 & 38.2 & 36.5 & 43.0 & 37.0 & 55.5 \\
\hline & mBERT-110k & 26.0 & 40.5 & 42.9 & 43.8 & 27.7 & 24.0 & 31.9 & 33.9 & 50.3 \\
& 30k & 38.5 & 28.8 & 29.8 & 33.9 & 38.9 & 26.4 & 34.1 & 45.8 & 42.7 \\
fastText & 120k & 51.6 & 48.9 & 55.2 & 49.7 & 54.1 & 44.1 & 54.8 & 56.0 & 60.9 \\
& 250k & 55.0 & 56.0 & 59.1 & 55.4 & 58.1 & 49.2 & 59.2 & 59.5 & 63.9 \\
& 500k & $\mathbf{5 7 . 0}$ & 59.1 & 61.5 & 58.0 & $\mathbf{5 9 . 2}$ & 50.9 & $\mathbf{5 9 . 7}$ & $\mathbf{6 1 . 0}$ & $\mathbf{6 4 . 6}$ \\
& 1000k & 56.4 & $\mathbf{6 0 . 7}$ & $\mathbf{6 2 . 2}$ & $\mathbf{5 9 . 1}$ & 58.9 & $\mathbf{5 1 . 7}$ & 57.5 & 57.2 & 63.7
\end{tabular}

Table 9: p5 for mBERT and fastText on mLAMA. Numbers across languages are not comparable as the number of triples varies.

\begin{tabular}{lrrrrrrrrrrr} 
& $\begin{array}{c}\text { Vocab. } \\
\text { Model }\end{array}$ & Size & AR & DE & ES & FI & HE & JA & KO & TH & TR \\
\hline \hline Oracle & & 23.1 & 23.8 & 23.2 & 22.9 & 24.5 & $\mathbf{2 2 . 5}$ & 22.6 & 25.1 & 24.6 \\
\hline mBERT & $110 \mathrm{k}$ & 12.1 & 26.1 & 27.6 & 15.8 & 11.0 & 11.8 & 15.1 & 10.8 & 27.7 \\
\hline & mBERT-110k & 7.8 & 14.3 & 16.9 & 15.0 & 6.6 & 6.4 & 8.0 & 7.4 & 19.4 \\
& 30k & 12.4 & 8.9 & 9.0 & 9.4 & 13.8 & 7.4 & 9.4 & 14.8 & 14.5 \\
fastText & $120 \mathrm{k}$ & 20.2 & 18.9 & 23.8 & 18.1 & 22.1 & 15.4 & 21.0 & 23.8 & 26.1 \\
& 250k & 22.7 & 24.0 & 27.3 & 22.6 & 26.3 & 18.0 & 23.8 & $\mathbf{2 8 . 3}$ & 28.7 \\
& 500k & $\mathbf{2 4 . 2}$ & 26.6 & $\mathbf{3 0 . 1}$ & 24.3 & 27.4 & 20.0 & $\mathbf{2 5 . 0}$ & 27.6 & 29.4 \\
& $1000 \mathrm{k}$ & 23.7 & $\mathbf{2 7 . 6}$ & $\mathbf{3 0 . 1}$ & $\mathbf{2 5 . 6}$ & $\mathbf{2 7 . 5}$ & 20.4 & 23.2 & 27.2 & $\mathbf{2 9 . 8}$
\end{tabular}

Table 10: p1 for mBERT and fastText on mLAMAUHN. Numbers across languages are not comparable as the number of triples varies. 


\begin{tabular}{|c|c|c|c|c|c|}
\hline Relation & Subject & Template & Object & BERT & fastText \\
\hline P1412 & William James & {$[\mathrm{X}]$ used to communicate in $[\mathrm{Y}]$. } & English & English & Irish \\
\hline P1412 & Bernardino Ochino & {$[\mathrm{X}]$ used to communicate in $[\mathrm{Y}]$. } & Italian & Spanish & Italian \\
\hline P1412 & Mick Lally & {$[\mathrm{X}]$ used to communicate in $[\mathrm{Y}]$. } & Irish & English & Irish \\
\hline P1412 & Robert Naunton & {$[\mathrm{X}]$ used to communicate in $[\mathrm{Y}]$. } & English & English & Welsh \\
\hline P108 & Steve Jobs & {$[\mathrm{X}]$ works for $[\mathrm{Y}]$} & Apple Inc. & Microsoft & Apple Inc. \\
\hline P108 & Steve Wozniak & {$[\mathrm{X}]$ works for $[\mathrm{Y}]$. } & Apple Inc. & CBS & Apple Inc. \\
\hline P108 & Grady Booch & {$[\mathrm{X}]$ works for $[\mathrm{Y}]$. } & IBM & IBM & Apple Inc. \\
\hline P108 & Philip Don Estridge & {$[\mathrm{X}]$ works for $[\mathrm{Y}]$. } & IBM & IBM & Apple Inc. \\
\hline P178 & Safari & {$[\mathrm{X}]$ is developed by $[\mathrm{Y}]$. } & Apple Inc. & Intel & Apple Inc. \\
\hline P178 & PostScript & {$[\mathrm{X}]$ is developed by $[\mathrm{Y}]$. } & Adobe & Microsoft & Adobe \\
\hline P178 & Active Directory & {$[\mathrm{X}]$ is developed by $[\mathrm{Y}]$. } & Microsoft & Microsoft & Apple Inc. \\
\hline P178 & Internet Explorer & {$[\mathrm{X}]$ is developed by $[\mathrm{Y}]$. } & Microsoft & Microsoft & Google \\
\hline P31 & Long Preston & {$[\mathrm{X}]$ is a $[\mathrm{Y}]$. } & village & village & pub \\
\hline P31 & Israfil & {$[\mathrm{X}]$ is a $[\mathrm{Y}]$. } & angel & village & angel \\
\hline P31 & alfuzosin & {$[\mathrm{X}]$ is a $[\mathrm{Y}]$. } & medication & protein & medication \\
\hline P31 & Crawfordsburn & {$[\mathrm{X}]$ is $\mathrm{a}[\mathrm{Y}]$. } & village & village & suburb \\
\hline P36 & Cook County & The capital of $[\mathrm{X}]$ is $[\mathrm{Y}]$. & Chicago & Chicago & Williamson \\
\hline P36 & Cayuga County & The capital of $[\mathrm{X}]$ is $[\mathrm{Y}]$. & Auburn & Auburn & Greenville \\
\hline P36 & Grand Est & The capital of $[\mathrm{X}]$ is $[\mathrm{Y}]$. & Strasbourg & Paris & Strasbourg \\
\hline P36 & Caddo Parish & The capital of $[\mathrm{X}]$ is $[\mathrm{Y}]$. & Shreveport & Georgetown & Shreveport \\
\hline P407 & The Vampyre & {$[\mathrm{X}]$ was written in $[\mathrm{Y}]$. } & English & English & Gothic \\
\hline P407 & Empire & {$[\mathrm{X}]$ was written in $[\mathrm{Y}]$. } & English & English & Persian \\
\hline P407 & Politika & {$[\mathrm{X}]$ was written in $[\mathrm{Y}]$. } & Serbian & Latin & Serbian \\
\hline P407 & Lenta.ru & {$[\mathrm{X}]$ was written in $[\mathrm{Y}]$. } & Russian & German & Russian \\
\hline P449 & Drake \& Josh & {$[\mathrm{X}]$ was originally aired on $[\mathrm{Y}]$. } & Nickelodeon & Nickelodeon & Fox Arena \\
\hline P449 & Salute Your Shorts & {$[\mathrm{X}]$ was originally aired on $[\mathrm{Y}]$. } & Nickelodeon & Nickelodeon & Lifetime \\
\hline P449 & Yo Momma & {$[\mathrm{X}]$ was originally aired on $[\mathrm{Y}]$. } & MTV & CBS & MTV \\
\hline P449 & Hey Arnold! & {$[\mathrm{X}]$ was originally aired on $[\mathrm{Y}]$. } & Nickelodeon & CBS & Nickelodeon \\
\hline P127 & Xbox & {$[\mathrm{X}]$ is owned by $[\mathrm{Y}]$. } & Microsoft & Microsoft & Nintendo \\
\hline P127 & Eiffel Tower & {$[\mathrm{X}]$ is owned by $[\mathrm{Y}]$. } & Paris & Boeing & Paris \\
\hline P127 & Lotus Software & {$[\mathrm{X}]$ is owned by $[\mathrm{Y}]$. } & IBM & IBM & Microsoft \\
\hline P127 & Lexus & {$[\mathrm{X}]$ is owned by $[\mathrm{Y}]$. } & Toyota & Chrysler & Toyota \\
\hline P364 & Black Narcissus & The original language of $[\mathrm{X}]$ is $[\mathrm{Y}]$. & English & English & Irish \\
\hline P364 & The God Delusion & The original language of $[\mathrm{X}]$ is $[\mathrm{Y}]$. & English & English & Hebrew \\
\hline P364 & Vecinos & The original language of $[\mathrm{X}]$ is $[\mathrm{Y}]$. & Spanish & Latin & Spanish \\
\hline P364 & Janji Joni & The original language of $[\mathrm{X}]$ is $[\mathrm{Y}]$. & Indonesian & Marathi & Indonesian \\
\hline P106 & Halle Berry & {$[\mathrm{X}]$ is a $[\mathrm{Y}]$ by profession. } & model & model & organist \\
\hline P106 & Gregory Chamitoff & {$[\mathrm{X}]$ is a $[\mathrm{Y}]$ by profession. } & astronaut & lawyer & astronaut \\
\hline P106 & Karl Taylor Compton & {$[\mathrm{X}]$ is a $[\mathrm{Y}]$ by profession. } & physicist & lawyer & physicist \\
\hline P106 & Herbert Romulus O'Conor & {$[\mathrm{X}]$ is a $[\mathrm{Y}]$ by profession . } & lawyer & lawyer & playwright \\
\hline P176 & System Controller Hub & {$[\mathrm{X}]$ is produced by $[\mathrm{Y}]$. } & Intel & Intel & Apple Inc. \\
\hline P176 & Daihatsu Boon & {$[\mathrm{X}]$ is produced by $[\mathrm{Y}]$. } & Toyota & Honda & Toyota \\
\hline P176 & British Rail Class 360 & {$[\mathrm{X}]$ is produced by $[\mathrm{Y}]$. } & Siemens & Siemens & Volvo Cars \\
\hline P176 & Dino & {$[\mathrm{X}]$ is produced by $[\mathrm{Y}]$. } & Ferrari & Sony & Ferrari \\
\hline P937 & Howard Florey & {$[\mathrm{X}]$ used to work in $[\mathrm{Y}]$. } & London & London & Montgomery \\
\hline P937 & Alberts Kviesis & {$[\mathrm{X}]$ used to work in $[\mathrm{Y}]$. } & Riga & Stockholm & Riga \\
\hline P937 & Ramsay MacDonald & {$[\mathrm{X}]$ used to work in $[\mathrm{Y}]$. } & London & London & Scotland \\
\hline P937 & Juan March & {$[\mathrm{X}]$ used to work in $[\mathrm{Y}]$. } & Madrid & Paris & Madrid \\
\hline P463 & United States of America & {$[\mathrm{X}]$ is a member of $[\mathrm{Y}]$. } & NATO & NATO & PBS \\
\hline P463 & Croatia & {$[\mathrm{X}]$ is a member of $[\mathrm{Y}]$. } & NATO & NATO & FIFA \\
\hline P463 & Mexico national football team & {$[\mathrm{X}]$ is a member of $[\mathrm{Y}]$. } & FIFA & CONCACAF & FIFA \\
\hline P463 & Estonia & {$[\mathrm{X}]$ is a member of $[\mathrm{Y}]$. } & NATO & FIFA & NATO \\
\hline P138 & Germany & {$[\mathrm{X}]$ is named after $[\mathrm{Y}]$. } & Bavaria & France & Bavaria \\
\hline P138 & GNU & {$[\mathrm{X}]$ is named after $[\mathrm{Y}]$. } & Unix & Aristotle & Unix \\
\hline P138 & solar mass & {$[\mathrm{X}]$ is named after $[\mathrm{Y}]$. } & Sun & Sun & carbon \\
\hline P138 & Torino F.C. & {$[\mathrm{X}]$ is named after $[\mathrm{Y}]$. } & Turin & Turin & Apple Inc. \\
\hline P101 & Edward Burnett Tylor & {$[\mathrm{X}]$ works in the field of $[\mathrm{Y}]$. } & anthropology & medicine & anthropology \\
\hline P101 & Anaxagoras & {$[\mathrm{X}]$ works in the field of $[\mathrm{Y}]$. } & philosophy & philosophy & philosopher \\
\hline P101 & Adam Carolla & {$[\mathrm{X}]$ works in the field of $[\mathrm{Y}]$. } & comedian & psychology & comedian \\
\hline P101 & physical system & {$[\mathrm{X}]$ works in the field of $[\mathrm{Y}]$. } & physics & physics & physiology \\
\hline P39 & Augustine Kandathil & {$[\mathrm{X}]$ has the position of $[\mathrm{Y}]$. } & archbishop & minister & archbishop \\
\hline P39 & John XXI & {$[\mathrm{X}]$ has the position of $[\mathrm{Y}]$. } & pope & bishop & pope \\
\hline P39 & Photinus of Sirmium & {$[\mathrm{X}]$ has the position of $[\mathrm{Y}]$. } & bishop & bishop & pope \\
\hline P39 & Samson of Dol & {$[\mathrm{X}]$ has the position of $[\mathrm{Y}]$. } & bishop & bishop & God \\
\hline P530 & Holy See & {$[\mathrm{X}]$ maintains diplomatic relations with $[\mathrm{Y}]$. } & Italy & Italy & Austria \\
\hline P530 & Malta & {$[\mathrm{X}]$ maintains diplomatic relations with $[\mathrm{Y}]$. } & Italy & Italy & Malta \\
\hline P530 & Liechtenstein & {$[\mathrm{X}]$ maintains diplomatic relations with [Y] } & Austria & Switzerland & Austria \\
\hline P530 & Saudi Arabia & {$[\mathrm{X}]$ maintains diplomatic relations with $[\mathrm{Y}]$. } & Kuwait & Qatar & Kuwait \\
\hline P264 & Georg Solti & {$[\mathrm{X}]$ is represented by music label $[\mathrm{Y}]$. } & Decca & EMI & Decca \\
\hline P264 & The Temptations & {$[\mathrm{X}]$ is represented by music label $[\mathrm{Y}]$. } & Motown & EMI & Motown \\
\hline P264 & David Bowie & {$[\mathrm{X}]$ is represented by music label $[\mathrm{Y}]$. } & EMI & EMI & Barclay \\
\hline P264 & Maria Callas & {$[\mathrm{X}]$ is represented by music label $[\mathrm{Y}]$. } & EMI & EMI & Decca \\
\hline P1376 & Florence & {$[\mathrm{X}]$ is the capital of $[\mathrm{Y}]$. } & Tuscany & Italy & Tuscany \\
\hline P1376 & Canberra & {$[\mathrm{X}]$ is the capital of $[\mathrm{Y}]$. } & Australia & Australia & Queensland \\
\hline P1376 & Heraklion & {$[\mathrm{X}]$ is the capital of $[\mathrm{Y}]$. } & Crete & Greece & Crete \\
\hline P1376 & Islamabad & {$[\mathrm{X}]$ is the capital of $[\mathrm{Y}]$. } & Pakistan & Pakistan & Karachi \\
\hline P1001 & Jatiya Sangshad & {$[\mathrm{X}]$ is a legal term in $[\mathrm{Y}]$. } & Bangladesh & India & Bangladesh \\
\hline P1001 & Legislative Yuan & {$[\mathrm{X}]$ is a legal term in $[\mathrm{Y}]$. } & Taiwan & Singapore & Taiwan \\
\hline P1001 & Manitoba Act, 1870 & {$[\mathrm{X}]$ is a legal term in $[\mathrm{Y}]$. } & Canada & Canada & Ontario \\
\hline P1001 & Yang di-Pertuan Agong & {$[\mathrm{X}]$ is a legal term in $[\mathrm{Y}]$. } & Malaysia & Malaysia & Brunei \\
\hline P495 & soppressata & {$[\mathrm{X}]$ was created in $[\mathrm{Y}]$. } & Italy & Italy & Peru \\
\hline P495 & Kefalotyri & {$[\mathrm{X}]$ was created in $[\mathrm{Y}]$. } & Greece & Cyprus & Greece \\
\hline P495 & Degrassi High & {$[\mathrm{X}]$ was created in $[\mathrm{Y}]$. } & Canada & Canada & Jordan \\
\hline P495 & Fox Soccer News & {$[\mathrm{X}]$ was created in $[\mathrm{Y}]$. } & Canada & Australia & Canada \\
\hline
\end{tabular}

Table 11: We sample two random triples where either BERT or fastText[1000k] is correct per relation. One can see for example that BERT mostly predicts "jazz" for relation P136. 


\begin{tabular}{|c|c|c|c|c|c|}
\hline Relation & Subject & Template & Object & BERT & fastText \\
\hline P527 & army & {$[\mathrm{X}]$ consists of $[\mathrm{Y}]$. } & infantry & infantry & cavalry \\
\hline P527 & Windward Islands & {$[\mathrm{X}]$ consists of $[\mathrm{Y}]$. } & Barbados & Bermuda & Barbados \\
\hline P527 & taxon & {$[\mathrm{X}]$ consists of $[\mathrm{Y}]$. } & organism & grass & organism \\
\hline P527 & humanities & {$[\mathrm{X}]$ consists of $[\mathrm{Y}]$. } & art & art & linguistics \\
\hline P1303 & Kenny G & {$[\mathrm{X}]$ plays $[\mathrm{Y}]$} & saxophone & guitar & saxophone \\
\hline P1303 & Stuart Duncan & {$[\mathrm{X}]$ plays $[\mathrm{Y}]$. } & fiddle & guitar & fiddle \\
\hline P1303 & Herbie Nichols & {$[\mathrm{X}]$ plays $[\mathrm{Y}]$. } & piano & piano & harmonica \\
\hline P1303 & Nat King Cole & {$[\mathrm{X}]$ plays $[\mathrm{Y}]$. } & piano & piano & saxophone \\
\hline P190 & Uzhhorod & {$[\mathrm{X}]$ and $[\mathrm{Y}]$ are twin cities. } & Moscow & Moscow & Lviv \\
\hline P190 & Vienna & {$[\mathrm{X}]$ and $[\mathrm{Y}]$ are twin cities . } & Budapest & Budapest & Vienna \\
\hline P190 & Cali & {$[\mathrm{X}]$ and $[\mathrm{Y}]$ are twin cities . } & Guadalajara & Santiago & Guadalajara \\
\hline P190 & Mindelo & {$[\mathrm{X}]$ and $[\mathrm{Y}]$ are twin cities. } & Porto & Santiago & Porto \\
\hline P47 & Monreale & {$[\mathrm{X}]$ shares border with $[\mathrm{Y}]$. } & Palermo & Italy & Palermo \\
\hline P47 & Afghanistan & {$[\mathrm{X}]$ shares border with $[\mathrm{Y}]$. } & Pakistan & Pakistan & Afghanistan \\
\hline P47 & Ukraine & {$[\mathrm{X}]$ shares border with $[\mathrm{Y}]$. } & Russia & Russia & Ukraine \\
\hline P47 & Edegem & {$[\mathrm{X}]$ shares border with $[\mathrm{Y}]$. } & Antwerp & Ethiopia & Antwerp \\
\hline P30 & McDonald Heights & {$[\mathrm{X}]$ is located in $[\mathrm{Y}]$. } & Antarctica & Africa & Antarctica \\
\hline P30 & Balham Valley & {$[\mathrm{X}]$ is located in $[\mathrm{Y}]$. } & Antarctica & Antarctica & Africa \\
\hline P30 & Southern Netherlands & {$[\mathrm{X}]$ is located in $[\mathrm{Y}]$. } & Europe & Europe & Africa \\
\hline P30 & Pitcairn Islands & {$[\mathrm{X}]$ is located in $[\mathrm{Y}]$. } & Oceania & Antarctica & Oceania \\
\hline P361 & arithmetic & {$[\mathrm{X}]$ is part of $[\mathrm{Y}]$. } & mathematics & mathematics & $\operatorname{logic}$ \\
\hline P361 & agricultural science & {$[\mathrm{X}]$ is part of $[\mathrm{Y}]$. } & agriculture & agriculture & science \\
\hline P361 & zoology & {$[\mathrm{X}]$ is part of $[\mathrm{Y}]$. } & biology & science & biology \\
\hline P361 & neuroscience & {$[\mathrm{X}]$ is part of $[\mathrm{Y}]$. } & psychology & science & psychology \\
\hline P103 & Muppalaneni Shiva & The native language of $[\mathrm{X}]$ is $[\mathrm{Y}]$. & Telugu & Marathi & Telugu \\
\hline P103 & Joseph Reinach & The native language of $[\mathrm{X}]$ is $[\mathrm{Y}]$. & French & English & French \\
\hline P103 & Raymond Queneau & The native language of $[\mathrm{X}]$ is $[\mathrm{Y}]$. & French & French & Breton \\
\hline P103 & Lindsey Davis & The native language of $[\mathrm{X}]$ is $[\mathrm{Y}]$. & English & English & Welsh \\
\hline P20 & James Northcote & {$[\mathrm{X}]$ died in $[\mathrm{Y}]$. } & London & London & Morris \\
\hline P20 & George Frampton & {$[\mathrm{X}]$ died in $[\mathrm{Y}]$. } & London & London & Chapman \\
\hline P20 & Peter Strudel & {$[\mathrm{X}]$ died in $[\mathrm{Y}]$. } & Vienna & Paris & Vienna \\
\hline P20 & Gaetano Gandolfi & {$[\mathrm{X}]$ died in $[\mathrm{Y}]$. } & Bologna & Rome & Bologna \\
\hline P27 & August Gailit & {$[\mathrm{X}]$ is $[\mathrm{Y}]$ citizen . } & Estonia & Luxembourg & Estonia \\
\hline P27 & Ada Yonath & {$[\mathrm{X}]$ is $[\mathrm{Y}]$ citizen . } & Israel & India & Israel \\
\hline P27 & Enrique Llanes & {$[\mathrm{X}]$ is $[\mathrm{Y}]$ citizen . } & Mexico & Mexico & Spain \\
\hline P27 & Timothy Anglin & {$[\mathrm{X}]$ is $[\mathrm{Y}]$ citizen . } & Canada & Canada & England \\
\hline P279 & Ciliary neurotrophic factor & {$[\mathrm{X}]$ is a subclass of $[\mathrm{Y}]$. } & protein & protein & inflammation \\
\hline P279 & Decorin & {$[\mathrm{X}]$ is a subclass of $[\mathrm{Y}]$. } & protein & protein & perfume \\
\hline P279 & shinto shrine & {$[\mathrm{X}]$ is a subclass of $[\mathrm{Y}]$. } & sanctuary & Buddhism & sanctuary \\
\hline P279 & articled clerk & {$[\mathrm{X}]$ is a subclass of $[\mathrm{Y}]$. } & apprentice & jurist & apprentice \\
\hline P19 & Frans Floris I & {$[\mathrm{X}]$ was born in $[\mathrm{Y}]$. } & Antwerp & Amsterdam & Antwerp \\
\hline P19 & Sajjad Ali & {$[\mathrm{X}]$ was born in $[\mathrm{Y}]$. } & Lahore & Tehran & Lahore \\
\hline P19 & Henry Mayhew & {$[\mathrm{X}]$ was born in $[\mathrm{Y}]$. } & London & London & Fowler \\
\hline P19 & Rob Lee & {$[\mathrm{X}]$ was born in $[\mathrm{Y}]$. } & London & London & Gary \\
\hline P159 & Swedish Orphan Biovitrum & The headquarter of $[\mathrm{X}]$ is in $[\mathrm{Y}]$. & Stockholm & Stockholm & Gothenburg \\
\hline P159 & Canadian Jewish Congress & The headquarter of $[\mathrm{X}]$ is in $[\mathrm{Y}]$. & Ottawa & Ottawa & Winnipeg \\
\hline P159 & Florida International University & The headquarter of $[\mathrm{X}]$ is in $[\mathrm{Y}]$. & Miami & Tampa & Miami \\
\hline P159 & Edipresse & The headquarter of $[\mathrm{X}]$ is in $[\mathrm{Y}]$. & Lausanne & Chennai & Lausanne \\
\hline P413 & Markus Halsti & {$[\mathrm{X}]$ plays in $[\mathrm{Y}]$ position } & midfielder & midfielder & goaltender \\
\hline P413 & Luca Danilo Fusi & {$[\mathrm{X}]$ plays in $[\mathrm{Y}]$ position.} & midfielder & midfielder & goalkeeper \\
\hline P413 & Mike Teel & {$[\mathrm{X}]$ plays in $[\mathrm{Y}]$ position.} & quarterback & forward & quarterback \\
\hline P413 & Doug Buffone & {$[\mathrm{X}]$ plays in $[\mathrm{Y}]$ position.} & linebacker & forward & linebacker \\
\hline P37 & Sorengo & The official language of $[\mathrm{X}]$ is $[\mathrm{Y}]$. & Italian & Portuguese & Italian \\
\hline P37 & Padasjoki & The official language of $[\mathrm{X}]$ is $[\mathrm{Y}]$. & Finnish & English & Finnish \\
\hline P37 & Wallonia & The official language of $[\mathrm{X}]$ is $[\mathrm{Y}]$. & French & French & Basque \\
\hline P37 & Biel/Bienne & The official language of $[\mathrm{X}]$ is $[\mathrm{Y}]$. & French & French & Czech \\
\hline P140 & Gautama Buddha & {$[\mathrm{X}]$ is affiliated with the $[\mathrm{Y}]$ religion . } & Buddhism & Hindu & Buddhism \\
\hline P140 & Christianization & {$[\mathrm{X}]$ is affiliated with the $[\mathrm{Y}]$ religion. } & Christianity & Christian & Christianity \\
\hline P140 & Albanians & {$[\mathrm{X}]$ is affiliated with the $[\mathrm{Y}]$ religion . } & Christian & Christian & Muslim \\
\hline P740 & SNCF & {$[\mathrm{X}]$ was founded in $[\mathrm{Y}]$. } & Paris & Paris & France \\
\hline P740 & Odex & {$[\mathrm{X}]$ was founded in $[\mathrm{Y}]$. } & Singapore & Germany & Singapore \\
\hline P740 & Comerica & {$[\mathrm{X}]$ was founded in $[\mathrm{Y}]$. } & Detroit & Prague & Detroit \\
\hline P740 & Pink Fairies & {$[\mathrm{X}]$ was founded in $[\mathrm{Y}]$. } & London & London & Gold \\
\hline P276 & Saint-Domingue expedition & {$[\mathrm{X}]$ is located in $[\mathrm{Y}]$. } & Haiti & France & Haiti \\
\hline P276 & 2002 Australian $\mathrm{Op}[\mathrm{X}]$ is located in $[\mathrm{Y}]$. & Melbourne & Melbourne & Australia & \\
\hline P276 & 2013 German federal election & {$[\mathrm{X}]$ is located in $[\mathrm{Y}]$. } & Germany & Berlin & Germany \\
\hline P276 & Cantabrian Wars & {$[\mathrm{X}]$ is located in $[\mathrm{Y}]$. } & Spain & Spain & Catalonia \\
\hline P136 & Giulio Caccini & {$[\mathrm{X}]$ plays $[\mathrm{Y}]$ music } & opera & jazz & opera \\
\hline P136 & Nicolas Dalayrac & {$[\mathrm{X}]$ plays $[\mathrm{Y}]$ music . } & opera & jazz & opera \\
\hline P136 & Georgie Auld & {$[\mathrm{X}]$ plays $[\mathrm{Y}]$ music . } & jazz & jazz & ballad \\
\hline P136 & Chess Records & {$[\mathrm{X}]$ plays $[\mathrm{Y}]$ music . } & jazz & jazz & reggae \\
\hline P17 & Eibenstock & {$[\mathrm{X}]$ is located in $[\mathrm{Y}]$. } & Germany & Germany & Austria \\
\hline P17 & Vrienden van het Platteland & {$[\mathrm{X}]$ is located in $[\mathrm{Y}]$. } & Netherlands & Belgium & Netherlands \\
\hline P17 & Fawkner & {$[\mathrm{X}]$ is located in $[\mathrm{Y}]$. } & Australia & Lebanon & Australia \\
\hline P17 & Wakefield Park & {$[\mathrm{X}]$ is located in $[\mathrm{Y}]$. } & Australia & Australia & The Bahamas \\
\hline P131 & Squantz Pond State Park & {$[\mathrm{X}]$ is located in $[\mathrm{Y}]$. } & Connecticut & Somerset & Connecticut \\
\hline P131 & Ballyfermot & {$[\mathrm{X}]$ is located in $[\mathrm{Y}]$. } & Dublin & Ireland & Dublin \\
\hline P131 & Downtown East Village, Calgary & {$[\mathrm{X}]$ is located in $[\mathrm{Y}]$. } & Alberta & Alberta & Toronto \\
\hline P131 & Edmonton City Centre Airport & {$[\mathrm{X}]$ is located in $[\mathrm{Y}]$. } & Alberta & Alberta & Toronto \\
\hline
\end{tabular}

Table 12: Table 11 continued. 